\title{
Metamagnetic Shape Memory Alloy Thin Plates Consolidated by Compression Shearing Method at Room Temperature for Thermal Energy Harvesting Device
}

\author{
Hiroyuki Miki, ${ }^{1 *}$ Eijiro Abe, ${ }^{1,2}$ Sho Takeda, ${ }^{1}$ Makoto Ohtsuka, ${ }^{3}$ and Manfred Kohl ${ }^{4}$ \\ ${ }^{1}$ Institute of Fluid Science (IFS), Tohoku University, Katahira 2-1-1, Aoba-ku, Sendai, Miyagi 980-8577, Japan \\ ${ }^{2}$ Division of Mechanical Engineering, Graduate School of Engineering, Tohoku University, \\ Aramaki aza Aoba 6-3, Aoba-ku, Sendai, Miyagi 980-8578, Japan \\ ${ }^{3}$ Institute of Multidisciplinary Research for Advanced Materials (IMRAM), Tohoku University, \\ Katahira 2-1-1, Aoba-ku, Sendai, Miyagi 980-8577, Japan \\ ${ }^{4}$ Institute of Microstructure Technology (IMT), Karlsruhe Institute of Technology (KIT), \\ Postfach 3640, Karlsruhe 76021, Germany
}

(Received May 7, 2020; accepted July 22, 2020)

Keywords: metamagnetic material, shape memory alloy, severe plastic deformation, energy harvesting

As a powder metallurgy technique, the compression shearing method at room temperature (COSME-RT) is used for making metamagnetic shape memory alloy (MSMA) thin plates. COSME-RT is a method for consolidating a thin plate from powder raw material by a certain dynamic process that simultaneously applies a compression stress and a shearing strain to a powder material at room temperature and in ambient atmosphere. A plate of $50 \mu \mathrm{m}$ thickness fabricated by COSME-RT using Ni-Co-Mn-In powder from ground sputtered film is cut into 2 $\times 2 \times 0.05 \mathrm{~mm}^{3}$ and then applied to a thermal energy harvesting device. This device generates an average power density of $68 \mu \mathrm{W} \cdot \mathrm{cm}^{-3}$ and a peak power of $0.95 \mathrm{~mW} \cdot \mathrm{cm}^{-3}$, which makes it possible to supply a small amount of electric power to a certain type of sensor or small sensor device.

\section{Introduction}

As shape memory alloys, metamagnetic shape memory alloys (MSMAs) are expected to be used for microactuators because of their multifunctionality, similar to the application of ferromagnetic shape memory alloy actuators. ${ }^{(1-5)}$ MSMAs show a characteristic magnetic structural phase transition from a ferromagnetic austenite phase (A phase) to a ferrimagnetic or antiferromagnetic (weak magnetic) martensitic phase (M phase). ${ }^{(6-12)}$ Since this phase transition involves a large change in magnetization $(\Delta M)$, it is considered possible to apply MSMAs to actuators such as sensors and power generation devices that use a phase transition as the driving force.

Most of the conventional research on magnetic shape memory alloys including MSMAs has been related to bulk materials, and magnetostriction that is large enough for practical use has

*Corresponding author: e-mail: hiroyuki.miki.c2@tohoku.ac.jp

https://doi.org/10.18494/SAM.2020.2938 
been confirmed in Ni-Mn-Ga single-crystal samples but only under a strong applied magnetic field. ${ }^{(13)}$ Sputtering is one of the methods used for preparing an MSMA thin film. ${ }^{(14-17)}$ Sputtering makes it possible to fabricate thin films with a thickness of several microns, which is difficult with bulk materials. In addition, it is expected to contribute to improving the response in actuation and the downsizing of actuators. In previous research, it was confirmed that MSMA thin films show large changes in magnetization, a narrow temperature hysteresis, and a steep magnetic phase transition. ${ }^{(18,19)}$ However, when mounted on an actuator, sufficient material strength and output for practical use cannot be obtained even with a film thickness of several microns. To obtain them, a film with a thickness of several tens of microns is required, but this is not achieved by simply increasing the sputtering time, which causes a new and fundamental problem that very fast erosion of the target occurs. As one approach to solve this problem, Gueltig et al. proposed to solve the problems of material strength and output by stacking MSMA sputtered films, and made it possible to apply such films to an energy harvesting device that uses exhaust heat. ${ }^{(18,20)}$ However, since the sputtered films are attached with an adhesive to form a thick film, the thermal conductivity is poor compared with that of the bulk material. To obtain a large output in an energy harvesting device, a thin plate material of MSMA with a thickness of several tens of microns is required, and a new thin plate material manufacturing method that replaces the sputtering method has been required.

To address this issue, our research group focused on the compression shearing method at room temperature (COSME-RT) proposed by Takeishi et al. ${ }^{(21)}$ This method enables metal and magnetic material raw powders to be consolidated into plate-shaped bulk materials, and it is possible to efficiently fabricate thin plates from MSMA powder produced by crushing sputtered films. Conventional techniques such as heat treatment and addition of alloying elements have been used to improve shape memory properties and magnetic properties, ${ }^{(22)}$ but we also expect improvements in mechanical properties by a severe plastic deformation process.

In this study, we clarify the magnetic properties, phase transition temperature, and mechanical properties of an MSMA plate consolidated by COSME-RT to a thickness of $50 \mu \mathrm{m}$ and apply it to an energy harvesting device driven by the magnetic structural phase transition.

\section{Material Preparation}

First, a Ni-Co-Mn-In alloy sputtered film of $5 \mu \mathrm{m}$ thickness was prepared by the dualsource co-sputtering method using a Ni-Mn-In target and a Co target. The sputtering power for the Ni-Mn-In alloy target was $200 \mathrm{~W}$, and the sputtering power for the Co target was $8 \mathrm{~W}$. By adding Co to Ni-based Heusler alloys, the Curie temperature increases so as to induce the phase transition near room temperature, ${ }^{(23-25)}$ and the actuator can be driven in this temperature range. The Ni-Co-Mn-In film was sputtered on a polyvinyl alcohol substrate, and a freestanding film was obtained by peeling it from the substrate after deposition. Details of the sputtering process are given elsewhere. ${ }^{(18,19)}$ The peeled film was crushed using a mortar to obtain MSMA powder for COSME-RT.

The procedure for consolidating the alloy sheet material using COSME-RT ${ }^{(21)}$ apparatus (DRD-NNK-002, DIP Co., Ltd., Japan) is as follows. As the first step, the crushed powder was 
filled in a $20 \times 10 \mathrm{~mm}^{2}$ mold between a fixed plate and a moving plate in an air atmosphere. After filling, the powder sample was held under compressive stress $\sigma_{\mathrm{N}}$ and then the moving plate was displaced to apply shear strain $\gamma\left(=L_{\mathrm{S}} / T_{\mathrm{P}}\right)$ to the sample to solidify the thin plate. Figure 1 shows a schematic diagram of the consolidation process. Here, $L_{S}$ is the shear distance $(\mathrm{mm})$ and $T_{\mathrm{P}}$ is the sample thickness $(\mathrm{mm})$. The consolidation conditions for the target size of $20 \times 10 \times 0.05 \mathrm{~mm}^{3}$ plate are as follows: the raw powder weight is $0.08 \mathrm{~g}, \sigma_{\mathrm{N}}=2.5 \mathrm{GPa}$, $L_{\mathrm{S}}=0.1 \mathrm{~mm}, \gamma=2$, and the shear rate is $5 \mathrm{~mm} / \mathrm{min}$. After processing, the molded alloy plate was heat-treated at $1223 \mathrm{~K}$ for $3.6 \mathrm{ks}$ to improve the degree of regularity and crystallization.

\section{Material Properties}

Inductively coupled plasma-optical emission spectroscopy was carried out for composition analysis of the specimen. As a result, the composition of the heat-treated Ni-Co-Mn-In alloy plate consolidated by COSME-RT was identified as $\mathrm{Ni}_{47.7} \mathrm{Co}_{3.5} \mathrm{Mn}_{36.6} \mathrm{In}_{12.2}$.

Figure 2(a) shows the X-ray diffraction (XRD) profile of the MSMA plate after heat treatment obtained with $\mathrm{Cu}-\mathrm{Ka}$ radiation $(\lambda=1.5418 \AA)$ at room temperature. It is known from previous studies that the Ni-Co-Mn-In alloy has a Heusler-type crystal structure, and the crystal structure changes from the weak magnetic $\mathrm{M}$ phase to the ferromagnetic A phase with the magnetic structural phase transition. ${ }^{(6)}$ For an actuator that works at room temperature, a phase transition at room temperature or higher is required. ${ }^{(18,20)}$ In that case, it is necessary to

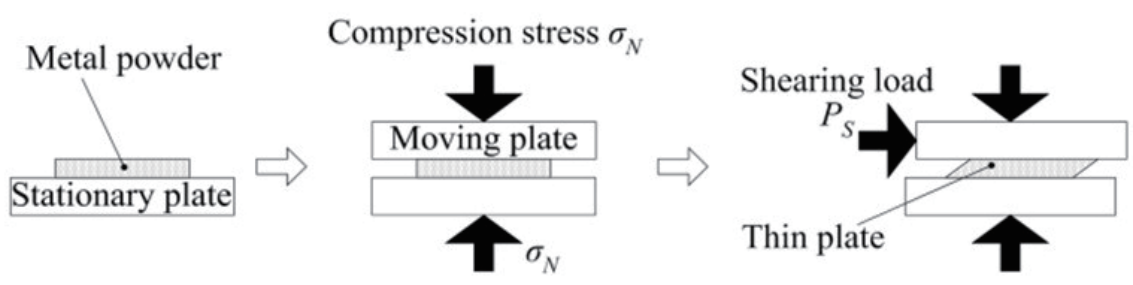

Fig. 1. Schematic diagram of the COSME-RT process. ${ }^{(21)}$

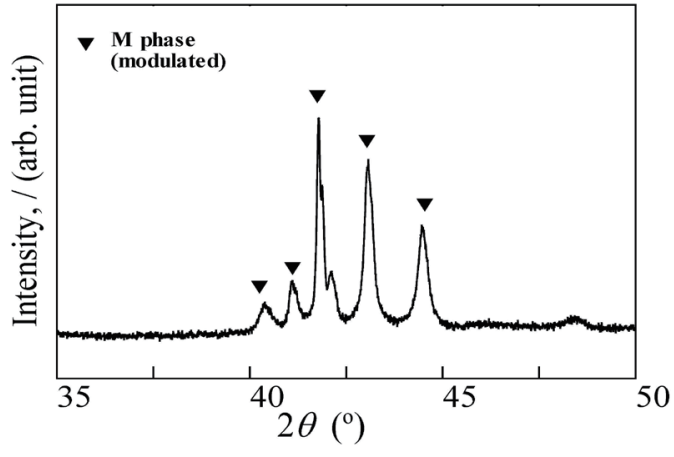

(a)

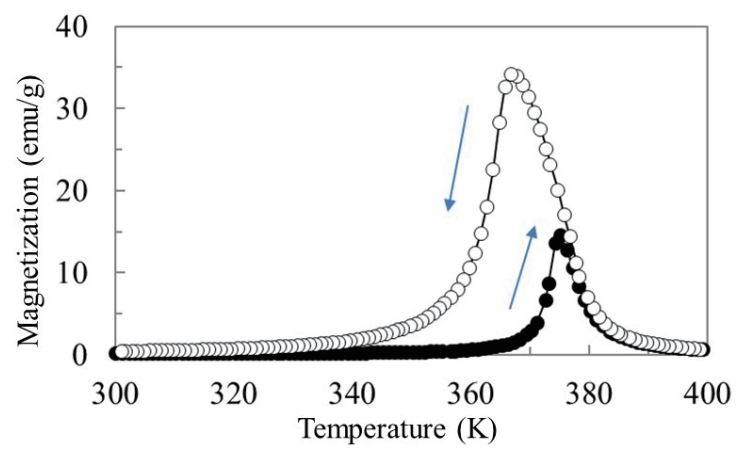

(b)

Fig. 2. (a) XRD profile and (b) temperature dependence of magnetization (M-T) curve at an external magnetic field of $0.05 \mathrm{~T}$ for annealed Ni-Co-Mn-In plate. 
confirm the diffraction peak of the M phase in XRD at room temperature. Diffraction peaks due to the long-period martensitic structure (14M) were confirmed around 40 to $45^{\circ}$ in Fig. 2(a). Therefore, it is confirmed that the structural phase transition of this material is above room temperature.

Next, differential scanning calorimetry analysis was performed to confirm the martensitic transformation temperature. The martensitic transformation start and end temperatures $M_{\mathrm{s}}$ and $M_{\mathrm{f}}$ were 365 and $350 \mathrm{~K}$, and the reverse martensitic transformation start and end temperatures $A_{\mathrm{s}}$ and $A_{\mathrm{f}}$ were 365 and $375 \mathrm{~K}$, respectively. The latent heat during the martensitic transformation and the inverse martensitic transformation were both $19 \mathrm{~J} / \mathrm{g}$.

The thermal magnetization curve of the heat-treated Ni-Co-Mn-In plate at $0.05 \mathrm{~T}$ is shown in Fig. 2(b). Here, a drastic change in the magnetization appears with the phase transformation, and the magnetization, which almost disappears in the $M$ phase, becomes a maximum of approximately $35 \mathrm{emu} / \mathrm{g}$ in the A phase. In the case of sputtered films with the same composition, the maximum magnetization is $26 \mathrm{emu} / \mathrm{g} ;{ }^{(18)}$ our alloy sheet has a magnetization $35 \%$ higher than this value. Also, from this magnetization curve, the Curie temperature $\left(T_{\mathrm{c}}\right)$ is $384 \mathrm{~K} ; M_{\mathrm{s}}, M_{\mathrm{f}}, A_{\mathrm{s}}$, and $A_{\mathrm{f}}$ are $366,356,368$, and $373 \mathrm{~K}$, respectively, and the structural phase transition occurred at temperatures above room temperature. Furthermore, the width of the temperature hysteresis became narrow (less than $10 \mathrm{~K}$ ).

\section{Thermal Energy Harvesting Device}

Figure 3 shows the principle of the energy harvesting operation of the actuator and Fig. 4 shows a photograph of the actual power generation device. The size of the MSMA plate prepared by COSME-RT was $2 \times 2 \times 0.05 \mathrm{~mm}^{3}$, and the cantilever supporting the coil and

(a)

(d)

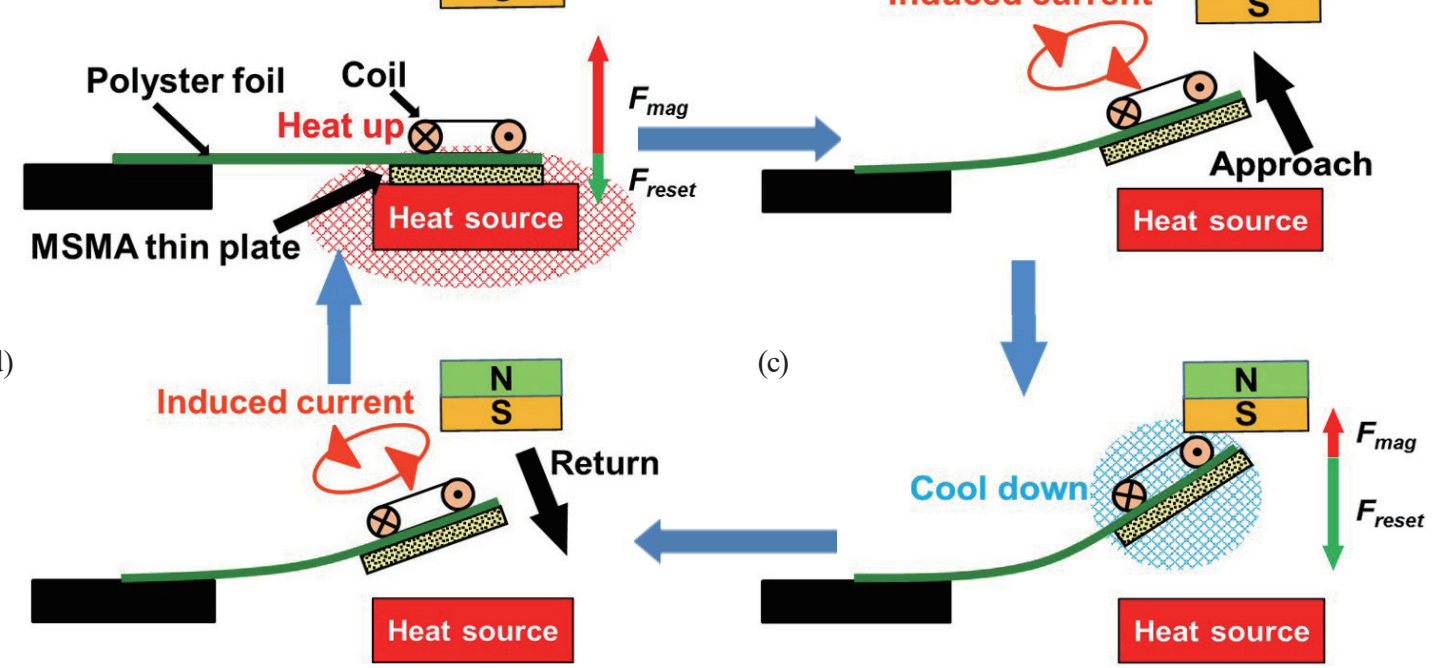

(b)

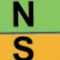

(1)

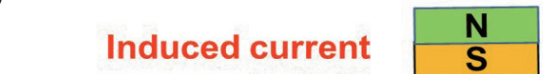

Fig. 3. (Color online) Operation principle of thermal energy harvesting device using an MSMA thin plate, (a) step 1: upper left, (b) step 2: upper right, (c) step 3: lower right, and (d) step 4: lower left. 


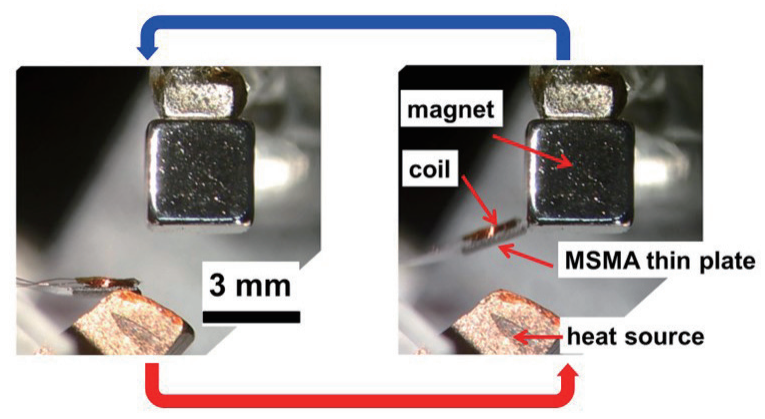

Fig. 4. (Color online) Thermal energy harvesting demonstrator device using a MSMA thin plate.

MSMA plate was a polyester film of $2 \times 5 \times 0.023 \mathrm{~mm}^{3}$. The MSMA plate was attached to the end of a polyester cantilever. In addition, a small coil was installed on the back side of the MSMA plate to collect the power. The small coil was designed to have inner and outer diameters of 1.5 and $3 \mathrm{~mm}$, respectively, a $\mathrm{Cu}$ wire with $15 \mu \mathrm{m}$ diameter was used, and the number of coil turns was 100. The polyester cantilever was set with one end fixed to the ceramic base plate and the other end free to move. The temperature of the heat source was set to $403 \mathrm{~K}\left(130{ }^{\circ} \mathrm{C}\right)$ because it is possible to increase the cooling efficiency during air cooling when the temperature difference from room temperature is sufficiently high.

When the plate is in contact with the heat source, as shown in Fig. 3(a), the inverse martensitic transformation to the ferromagnetic A phase occurs as the temperature and the magnetization increase; thus, the attractive force $F_{m a g}$ of the permanent magnet increases. At this time, $F_{\text {mag }}$ becomes larger than the elastic force $F_{\text {reset }}$ of the cantilever, and the tip of the cantilever approaches the magnet [Fig. 3(b)]. By moving it away from the heat source and toward the permanent magnet, the MSMA plate is cooled to below the martensitic transformation temperature and its magnetization decreases [Fig. (3c)]. In Fig. 3(c), $F_{\text {reset }}$ exceeds $F_{\text {mag }}$, and the tip of the cantilever moves away from the magnet to return to above the heat source [Fig. 3(d)]. These movements are repeated cyclically and result in continuous actuation movements. At this time, since the tip of the cantilever continues to move in the magnetic field gradient generated by the permanent magnet, an induced current can be continuously generated inside the coil in accordance with Faraday's law.

In this study, $\mathrm{NdFeB}$ magnets of $2 \times 2 \times 0.05 \mathrm{~mm}^{3}$ (small) and $3 \times 3 \times 3 \mathrm{~mm}^{3}$ (large) were prepared in order to change the magnetic field gradient generated near the tip of the cantilever. The device characteristics were evaluated.

\section{Results and Discussion}

\subsection{Estimate of acting force}

The magnetic attractive force $F_{\text {mag }}$ of the permanent magnet acting on the MSMA plate and the elastic reset force $F_{\text {reset }}$ of the polyester cantilever are calculated for the following states: the MSMA plate directly under the permanent magnet and on the heat source [Fig. 5(a)], and 


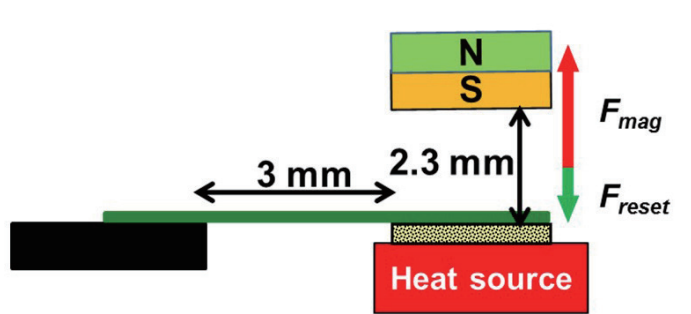

(a)

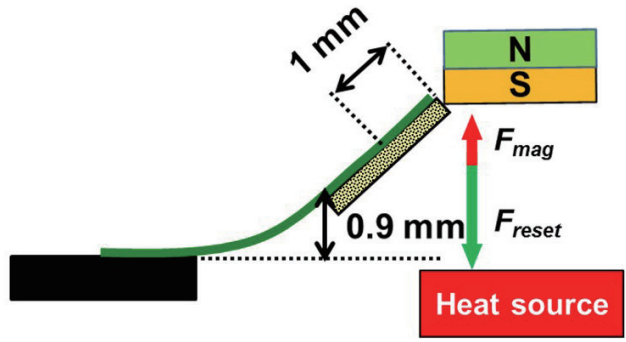

(b)

Fig. 5. (Color online) Schematic image for calculation in the condition of (a) MSMA plate on the heat source and (b) plate near a magnet.

the MSMA plate attracted to the permanent magnet and in contact with the magnet as shown in Fig. 5(b). For the calculation of $F_{m a g}$,

$$
F_{\text {mag }}=\mu_{0} q H
$$

was used. The parameters $q$ and $H$ represent the magnetic fields created by the magnetic poles of the MSMA plate and permanent magnets, respectively, and $H$ is described using the magnetic flux density $B$,

$$
B=H .
$$

Furthermore, the magnetic pole $q$ is calculated by using the magnetization $M$ and the surface area $A$ of the MSMA plate as

$$
q=M A .
$$

The elastic force $F_{\text {reset }}$ is written as

$$
F_{\text {reset }}=\left(d-d_{0}\right) \frac{E \cdot w \cdot t^{3}}{4 \cdot l^{3}}
$$

where the parameters $E, w, t, L, d$, and $d_{0}$ are the elastic modulus, plate width, plate thickness, beam length, the distance of the actual beam position from the magnet, and the distance from the magnet in the initial position, respectively. The MSMA plate at the tip was regarded as a rigid body, and the polyester cantilever was assumed to generate the reset force due to the deformation of the beam in the elastic region. The magnetization in the state is considered to be extremely small $(1 \mathrm{emu} / \mathrm{g})$. From Table 1 , it is confirmed that $F_{\text {mag }}$ exceeds $F_{\text {reset }}$ in the position shown in Fig. 5(a), and that the force required to approach the magnet is large. In contrast, in the state of Fig. 5(b), $F_{\text {reset }}$ exceeds $F_{\text {mag }}$, which confirms that the force required to return to the heat source works. 
Table 1

Magnetic parameters of demonstrator device and calculated forces.

\begin{tabular}{lcccc}
\hline $\begin{array}{l}\text { MSMA position } \\
\text { magnet }\end{array}$ & $\begin{array}{c}\text { On heat source } \\
\text { Small }\end{array}$ & $\begin{array}{c}\text { Nearby magnet } \\
\text { Large }\end{array}$ & $\begin{array}{c}\text { On heat source } \\
\text { Small }\end{array}$ & $\begin{array}{c}\text { Nearby magnet } \\
\text { Large }\end{array}$ \\
\hline Magnetization $M$ & $35 \mathrm{emu} / \mathrm{g}$ & $1 \mathrm{emu} / \mathrm{g}$ & $35 \mathrm{emu} / \mathrm{g}$ & $1 \mathrm{emu} / \mathrm{g}$ \\
Magnetic flux density $B$ & $20 \mathrm{mT}$ & $8 \mathrm{mT}$ & $90 \mathrm{mT}$ & $20 \mathrm{mT}$ \\
Force $F_{\text {mag }}$ & $22 \mathrm{mN}$ & $0.26 \mathrm{mN}$ & $101 \mathrm{mN}$ & $0.64 \mathrm{mN}$ \\
Force $F_{\text {reset }}$ & $0 \mathrm{mN}$ & $1.4 \mathrm{mN}$ & $0 \mathrm{mN}$ & $1.4 \mathrm{mN}$ \\
\hline
\end{tabular}

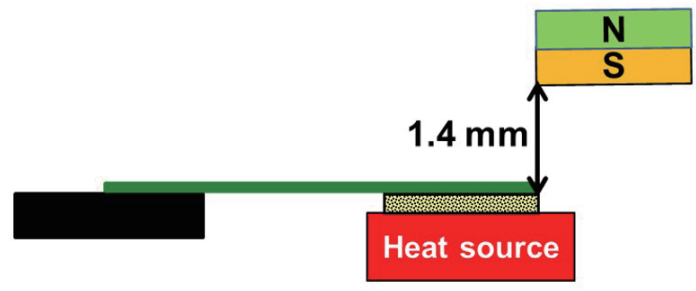

(a)

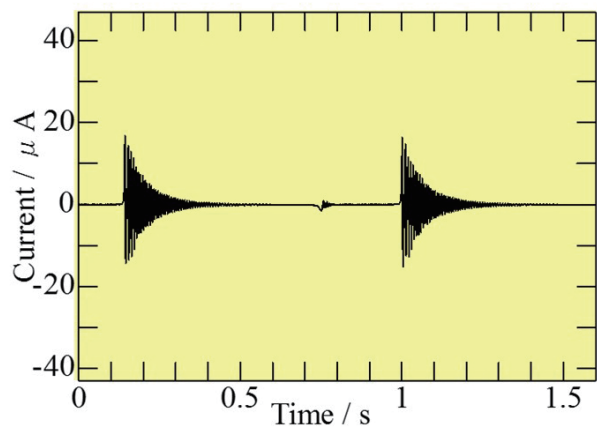

(b)

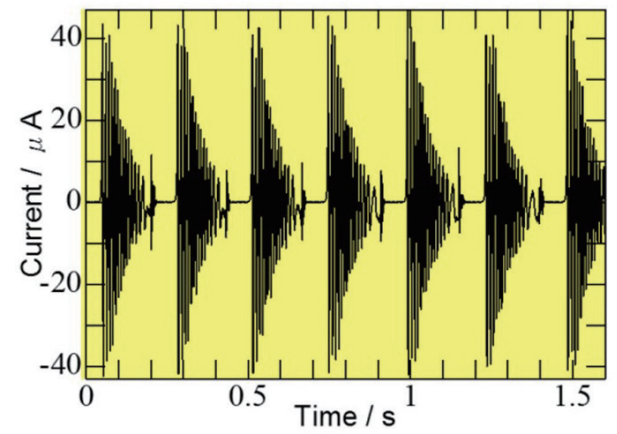

(c)

Fig. 6. (Color online) (a) Schematic diagram of demonstrator device. Time-resolved characteristic of induced current in the pick-up coil for the cases with the (b) small magnet and (c) large magnet.

\subsection{Evaluation of harvested power efficiency}

The following results were obtained in terms of the power generation efficiency of the prototype actuator device. As shown in Fig. 6(a), the maximum power density was obtained when the distance between the MSMA plate and the magnet was $1.4 \mathrm{~mm}$ and the end of the plate was directly below the opposite end of the magnet. The waveforms of the current generated in the coil when using the small magnet and the large magnet are shown in Figs. 6(b) and 6(c), respectively. From Fig. 6(b), when the small magnet is used, a maximum current of $16 \mu \mathrm{A}$ is generated, the power generation frequency is $1 \mathrm{~Hz}$, the average power density is $2.4 \mu \mathrm{W} \cdot \mathrm{cm}^{-3}$, and the maximum power density is $0.14 \mathrm{~mW} \cdot \mathrm{cm}^{-3}$. From Fig. 6(c), when the large magnet is used, a maximum current of $44 \mu \mathrm{A}$ is generated, the power generation frequency is $5 \mathrm{~Hz}$, the average power density is $68 \mu \mathrm{W} \cdot \mathrm{cm}^{-3}$, and the maximum power density is $0.95 \mathrm{~mW} \cdot \mathrm{cm}^{-3}$. When the small magnet was used, the maximum current was 1.6 times higher, the average power density was 1.5 times higher, and the maximum power density was 1.1 times higher than those 
when the thin films were laminated using an adhesive. ${ }^{(18)}$ On the other hand, in the case of the large magnet, the current value was 4.4 times higher, the average power density was 43 times higher, and the maximum power density was 7.3 times higher than those of laminated thin films.

\section{Conclusions}

We succeeded in consolidating a 50- $\mu$ m-thick MSMA plate using the COSME-RT method from a powder sample obtained by crushing a sputtered film. In addition, the consolidated MSMA plate showed a considerable change in magnetization along with the magnetic structural phase transition. This change in magnetization is $35 \%$ higher than that of a 5 - $\mu$ m-thick sputtered film. From the results of the magnetization curve, the transformation temperatures are $366,356,368$, and $373 \mathrm{~K}$ for $M_{\mathrm{s}}, M_{\mathrm{f}}, A_{\mathrm{s}}$, and $A_{\mathrm{f}}$, respectively, and the magnetic phase transition occurs at room temperature or higher with a narrow temperature hysteresis of about $10 \mathrm{~K}$. Furthermore, by applying this plate material to an energy harvesting device that uses a heat source of $403 \mathrm{~K}$, we were able to obtain a power generation capacity with an average power density of $68 \mu \mathrm{W} \cdot \mathrm{cm}^{-3}$ and a maximum power density of $0.95 \mathrm{~mW} \cdot \mathrm{cm}^{-3}$.

The proposed microactuator has a simple structure because it uses a natural cooling cycle. Therefore, it is useful for the power supply in an environment such as a plant or an engine where there is high-temperature exhaust heat but the installation space is limited. Considering a small wireless sensor as an application example, the required power is about $100 \mu \mathrm{W}$, ${ }^{(26)}$ and sufficient power can be supplied if the proposed device can output a power density of $1 \mu \cdot \mathrm{cm}^{-3}$ $\sim 1 \mathrm{~mW} \cdot \mathrm{cm}^{-3}$ as shown in this paper.

\section{Acknowledgments}

This work was partly supported by JSPS KAKENHI Grant Numbers $16 \mathrm{H} 04504$ and 19K22042. Extensive studies on COSME-RT by Prof. N. Nakayama and Dr. M. Horita of Shinshu University and Emeritus Prof. H. Takeishi of Chiba Institute of Technology are gratefully acknowledged.

\section{References}

1 M. Kohl, D. Brugger, M. Ohtsuka, and T. Takagi: Sens. Actuators, A 114 (2004) 445. https://doi.org/10.1016/ j.sna.2003.11.006

2 M. Kohl, D. Brugger, M. Ohtsuka, and T. Takagi: Sens. Actuators, A 135 (2007) 92. https://doi.org/10.1016/ j.sna.2006.08.014

3 M. Kohl, S. R. Yeduru, F. Khelfaoui, B. Krevet, A. Backen, S. Fähler, T. Eichhorn, G. Jakob, and A. Mecklenburg: Mat. Sci. Forum 635 (2010) 145. https://doi.org/10.4028/www.scientific.net/MSF.635.145

4 M. Kohl, M. Gueltig, V. Pinneker, R. Yin, F. Wendler, and B. Krevet: Micromachines 5 (2014) 1135. https://doi. org/10.3390/mi5041135

5 M. Gueltig, F. Wendler, H. Ossmer, M. Ohtsuka, H. Miki, T. Takagi, and M. Kohl: Adv. Energy Mater. 7 (2016) 1601879. https://doi.org/10.1002/aenm.201601879

6 Y. Sutou, Y. Imano, N. Koeda, T. Omori, R. Kainuma, K. Ishida, and K. Oikawa: Appl. Phys. Lett. 85 (2004) 4358. https://doi.org/10.1063/1.1808879 
7 K. Oikawa, W. Ito, Y. Imano, Y. Sutou, R. Kainuma, and K. Ishida: Appl. Phys. Lett. 88 (2006) 122507. https:// doi.org/10.1063/1.2187414

8 W. Ito, Y. Imano, R. Kainuma, Y. Sutou, K. Oikawa, and K. Ishida: Metall. Mat. Trans. A 38 (2007) 759. https://doi.org/10.1007/s11661-007-9094-9

9 W. Ito, M. Nagasako, R. Y. Umetsu, R. Kainuma, T. Kanomata, and K. Ishida: Appl. Phys. Lett. 93 (2008) 232503. https://doi.org/10.1063/1.3043456

10 S. Kustov, M. L. Corró, J. Pons, and E. Cesari: Appl. Phys. Lett. 94 (2009) 191901. https://doi. org/10.1063/1.3130229

11 S. Kustov, I. Golovin, M. L. Corró, and E. Cesari: J. Appl. Phys. 107 (2010) 053525. https://doi. org/10.1063/1.3313922

12 V. Recarte, J. I. Pérez-Landazábal, V. Sánchez-Alarcos, V. Zablotskii, E. Cesari, and S. Kustov: Acta Mater. 60 (2012) 3168. https://doi.org/10.1016/j.actamat.2012.02.022

13 A. Sozinov, A. A. Likhachev, N. Lanska, and K. Ullakko: Appl. Phys. Lett. 80 (2002) 1746. https://doi. org/10.1063/1.1458075

14 M. Suzuki, M. Ohtsuka, T. Suzuki, M. Matsumoto, and H. Miki: Mater. Trans. JIM 40 (1999) 1174. https://doi. org/10.2320/matertrans1989.40.1174

15 M. Ohtsuka, M. Sanada, M. Matsumoto, T. Takagi, and K. Itagaki: Mater. Trans. 44 (2003) 2513. https://doi. org/10.2320/matertrans.44.2513

16 M. Ohtsuka, Y. Konno, M. Matsumoto, T. Takagi, and K. Itagaki: Mater. Trans. 47 (2006) 625. https://doi. org/10.2320/matertrans.47.625

17 M. Ohtsuka, J. Sekino, K. Koyama, T. Takagi, and K. Itagaki: Eur. Phys. J. Spec. Top. 158 (2008) 173. https:// doi.org/10.1140/epjst/e2008-00672-4

18 M. Gueltig, H. Ossmer, M. Ohtsuka, H. Miki, K. Tsuchiya, T. Takagi, and M. Kohl: Adv. Energy Mater. 4 (2014) 4751. https://doi.org/10.1002/aenm.201400751

19 H. Miki, K. Tsuchiya, M. Ohtsuka, M. Gueltig, M. Kohl, and T. Takagi: Adv. Shape Memory Mater. 73 (2017) 149. https://doi.org/10.1007/978-3-319-53306-3_11

20 M. Gueltig, H. Ossmer, M. Ohtsuka, H. Miki, K. Tsuchiya, T. Takagi, and M. Kohl: Mater. Today Proc. 2 (2015) S883-S886. https://doi.org/10.1016/j.matpr.2015.07.423

21 H. Takeishi, N. Nakayama, and H. Miki: J. Soc. Mater. Sci. J. 54 (2005) 233. https://doi.org/10.2472/ jsms.54.233

22 R. Kainuma, Y. Imano, W. Ito, Y. Sutou, H. Morito, S. Okamoto, O. Kitakami, K. Oikawa, A. Fujita, T. Kanomata, and K. Ishida: Nature 439 (2006) 957. https://doi.org/10.1038/nature04493

23 V. Khovailo, T. Abe, V. Koledov, M. Matsumoto, H. Nakamura, R. Note, M. Ohtsuka, V. Shavrov, and T. Takagi: Mater. Trans. 44 (2003) 2509. https://doi.org/10.2320/matertrans.44.2509

24 V. Khovaylo, V. Koledov, V. Shavrov, M. Ohtsuka, H. Miki, T. Takagi, and V. Novosad: Metall. Mat. Trans. A 25 (2008) 322. https://doi.org/10.1016/j.msea.2006.12.235

25 K. Koike, M. Ohtsuka, M. Matsumoto, Y. Adachi, T. Takagi, and H. Kato: Eur. Phys. J. Spec. Top. 158 (2008) 143. https://doi.org/10.1140/epjst/e2008-00667-1

26 L. Tang, Y. Yang, and C. K. Soh: J. Intell. Mater. Syst. 21 (2010) 1867. https://doi. org/10.1177/1045389X10390249 